\title{
Soybean Lectin Agglutination
}

National Cancer Institute

\section{Source}

National Cancer Institute. Soybean Lectin Agglutination. NCI Thesaurus. Code C72902.

A technique for removing T lymphocytes based on differential agg lutination with soybean lectin. 